УДК 514.1

\title{
Группы движений собственно гельмгольцевой трехмерной геометрии и симплициальной трехмерной геометрии III типа
}

Р.А. Богданова, В.А. Кьров

Горно-Алтайский государственный университет (Горно-Алтайск, Россия)

\section{Groups of Motions of Properly Three-Dimensional Helmholtz Geometry and Simplical Three-Dimensional Geometry of the III Type}

\author{
R.A. Bogdanova, V.A. Kyrov \\ Gorno-Altaisk State University (Gorno-Altaisk, Russia)
}

\begin{abstract}
Основными задачами теории феноменологически симметричных (ФС) геометрий (геометрий локальной максимальной подвижности) являются их полная классификация, вывод уравнения феноменологической симметрии и нахождение групп движений для каждой из них. ФС геометрия задается на многообразии функцией пары точек. Феноменологическая симметрия трехмерных ФС геометрий состоит в наличии функциональной связи между значениями функции пары точек для всех пар из пяти произвольных точек. Их классификация была впервые построена B.Х. Левом и позже дополнена В.А. Кыровым симплициальной геометрией III типа. Методами установления групповой симметрии ФС геометрий являются метод решения функциональных уравнений на множество движений, разработанный для двумерных и некоторых трехмерных ФС геометрий, и метод экспоненциального отображения.

Методом экспоненциального отображения для собственно гельмгольцевой и симплициальной III типа трехмерных ФС геометрий находятся явные выражения групп движений. Данные вычисления производятся с использованием аппарата комплексного анализа и формулируются в виде отдельной теоремы. Группы движений этих геометрий являются действиями группы Ли $\mathrm{SL}_{2}(\mathrm{C})_{\mathrm{R}}$ в пространстве $\mathrm{R}^{3}$. Ключевые слова: геометрия максимальной подвижности, группа движений, группа Ли, алгебра Ли.
\end{abstract}

DOI 10.14258/izvasu(2019)4-10

Введение. В классификации трехмерных феноменологически симметричных геометрий (геометрий локальной максимальной подвижности) присутствуют собственно гельмгольцева геометрия и симплициальная геометрия III типа, которые задаются следующими функциями пары точек в $\mathrm{R}^{3}[1,2]$ :
The main tasks of the theory of phenomenologically symmetric (PS) geometries (geometries of local maximum mobility) are a complete classification of such geometries, derivation of the equation of phenomenological symmetry and finding groups of motions for the geometries. PS geometry is defined on a manifold by a function of a pair of points. Phenomenological symmetry of three-dimensional PS geometries lies in the presence of functional relation between the values of a pair of points for all pairs of five arbitrary points. Their classification was first built by V.Kh. Lev and later supplemented by V.A. Kyrov with simplicial type III geometry. Methods for establishing group symmetry of PS geometry contain a method of solving functional equations on a set of motions developed for two-dimensional and some three-dimensional PS geometries and the exponential mapping method.

This paper describes the process of finding explicit expressions for the groups of motions with the method of exponential mapping for the properly Helmholtz and simplicial type III three-dimensional PS geometries. These calculations are made using the apparatus of complex analysis and formulated as a separate theorem. These groups are actions of three Lie groups $\mathrm{SL}_{2}(\mathrm{C})_{\mathrm{R}}$ on the space $\mathrm{R}^{3}$.

Key words: maximal mobility geometry, group of motions, Lie group, Lie algebra.

$$
\begin{aligned}
& f(A, B)=\left(\left(x_{A}-x_{B}\right)^{2}+\left(y_{A}-y_{B}\right)^{2}\right) \times \\
& \quad \times \exp \left(2 \gamma \operatorname{arctg} \frac{y_{A}-y_{B}}{x_{A}-x_{B}}+w_{A}+w_{B}\right),
\end{aligned}
$$




$$
f(A, B)=\operatorname{arctg} \frac{y_{A}-y_{B}}{x_{A}-x_{B}}+w_{A}+w_{B},
$$

где $\left(x_{A}, y_{A}, w_{A}\right)$ и $\left(x_{B}, y_{B}, w_{B}\right)$ - координаты соответственно точек $A$ и $B$ в $R^{3}$.

Функции (1) и (2) перепишем, используя комплексные обозначения: $z=x+i y, \bar{z}=x-i y$, $\operatorname{arctg}(u)=\frac{1}{2 i} \operatorname{Ln} \frac{1+i u}{1-i u}, u^{a}=e^{a \operatorname{Ln} u}, i^{2}=-1$. Тогда для гельмогльцевой геометрии получаем

$$
\tilde{f}(A, B)=\frac{\left(\overline{z_{A}}-\overline{z_{B}}\right)^{\alpha}}{\left(z_{A}-z_{B}\right)} e^{u_{A}+u_{B}},
$$

где $\tilde{f}=f^{\frac{1}{i \gamma-1}}, w=u(i \gamma-1), \alpha=\frac{\gamma-i}{\gamma+i} \neq 1$, а для симплициальной геометрии III типа -

$$
\tilde{f}(A, B)=\frac{\overline{z_{A}}-\overline{z_{B}}}{z_{A}-z_{B}} e^{u_{A}+u_{B}}
$$

где $\tilde{f}=e^{-2 i f},-2 i w=u$.

Целью работы является нахождение явных выражений для действий групп Ли собственно гельмгольцевой трехмерной геометрии и симплициальной трехмерной геометрии III типа. Отметим, что об этих группах движений, без подробного доказательства, говорится в [2] (теореме 5).

Рассмотрим эффективное и дифференцируемое действие группы Ли $\mathrm{G}$ в $U \subset R^{3}$ [2-4], т.е. дифференцируемое отображение

$$
\lambda: U \times G \rightarrow U^{\prime},
$$

где $U^{\prime} \subset R^{3}$. Действие $\lambda_{a}$, определяемое произвольным элементом $a \in G$, называется движением пространства $R^{3}$ с функцией пары точек $f$, если для любых $A, B \in U$ таких, что $\langle A, B\rangle \in$ $S_{f},\left\langle\lambda_{a}(A), \lambda_{a}(B)\right\rangle \in S_{f}$, выполняется равенство

$$
f\left(\lambda_{a}(A), \lambda_{a}(B)\right)=f(A, B)
$$

причем $S_{f} \subseteq R^{3} \times R^{3}-$ область определения функции $f$.

Множество всех так определенных движений образует группу Ли движений. Ее алгебра Ли состоит из операторов $X=X^{1} \partial_{x}+X^{2} \partial_{y}+X^{3} \partial_{w}$, где $X^{\mu}=X^{\mu}(x, y, w)$ - дифференцируемая функция в $U, \mu=1,2,3[5]$.

Явный вид базисных операторов алгебр Ли групп движений собственно гельмгольцевой геометрии с функцией пары точек (1) и симплициальной геометрии III типа с функцией пары точек (2) найден в работе [6]. Запишем их в комплексном виде:

для собственно гельмгольцевой геометрии:

$$
\begin{aligned}
& \partial_{z}, \partial_{\bar{z}}, 2 z \partial_{z}+\partial_{u}, \\
& \quad-2 \bar{z} \partial_{\bar{z}}+\alpha \partial_{u}, z^{2} \partial_{z}+z \partial_{u},-\bar{z}^{2} \partial_{\bar{z}}+\alpha \bar{z} \partial_{u} ;
\end{aligned}
$$

для симплициальной геометрии III типа:

$$
\begin{aligned}
\partial_{z}, \partial_{\bar{z}}, 2 z \partial_{z}-\partial_{u}, & \\
& -2 \bar{z} \partial_{\bar{z}}-\partial_{u}, z^{2} \partial_{z}-z \partial_{u},-\bar{z}^{2} \partial_{\bar{z}}-\bar{z} \partial_{u} .
\end{aligned}
$$

В работе [2] доказано, что алгебры Ли (6) и (7) изоморфны алгебре Ли $s l_{2}(C)_{R}$, являющейся овеществлением комплексной алгебры Ли $s l_{2}(C)$ $[7,8]$.

Явные выражения для действий групп Ли. Метод нахождения групп движений заключается в применении экспоненциального отображения [9]:

$$
\left(\begin{array}{c}
x^{\prime} \\
y^{\prime} \\
w^{\prime}
\end{array}\right)=\operatorname{Exp}(t X)\left(\begin{array}{c}
x \\
y \\
w
\end{array}\right),
$$

где $t$ - вещественный параметр, а $X$ - произвольный оператор алгебры Ли группы движений,

$$
\operatorname{Exp}(t X)=1+t X+\frac{t^{2} X^{2}}{2 !}+\cdots
$$

С помощью экспоненциального отображения находятся однопараметрические подгруппы, соответствующие базисным операторам алгебр Ли групп движений, а затем композицией этих подгрупп ищутся явные выражения локальных действий групп Ли.

Поскольку базисные операторы (6) и (7) записаны в комплексном виде, то экспоненциальное отображение удобнее будет вычислить в комплексном виде:

$$
\left(\begin{array}{c}
z^{\prime} \\
\bar{z}^{\prime} \\
u^{\prime}
\end{array}\right)=\operatorname{Exp}(t Z)\left(\begin{array}{c}
z \\
\bar{z} \\
u
\end{array}\right),
$$

где $t$ - уже комплексный параметр, $Z$ - оператор алгебры Ли группы движений, являющийся линейной комбинацией операторов (6) или (7).

Теорема. Группы движений гельмгольцевой трехмерной геометрии с функиией пары точек (3) и симплициальной трехмерной геометрии III типа с функиией пары точек (4) в явном виде записываются так:

для собственно гельмгольщевой геометрии:

$$
\begin{aligned}
& z^{\prime}=\frac{a z+b}{c z+d}, \\
& u^{\prime}=u+\alpha \ln \overline{(c z+d)}-\ln (c z+d),
\end{aligned}
$$

для симплиииальной геометрии III типа:

$$
z^{\prime}=\frac{a z+b}{c z+d}, \quad u^{\prime}=\imath+\ln \frac{\overline{c z+d}}{c z+d}
$$

где $a d-b c=1, a, b, c, d=$ const $\in C$.

Доказательство. Сначала вычислим экспоненциальное отображение (8) для базисных операторов алгебры Ли (6). 
Применяя экспоненциальное отображение (8) к базисным операторам $Z_{1}=\partial_{z}, Z_{3}=2 z \partial_{z}+\partial_{u}$ и $Z_{5}=z^{2} \partial_{z}+z \partial_{u}$, получаем явные выражения подгрупп:

$$
\begin{aligned}
& \left(\begin{array}{c}
z^{\prime} \\
\bar{z}^{\prime} \\
u^{\prime}
\end{array}\right)=\operatorname{Exp}\left(t_{1} Z_{1}\right)\left(\begin{array}{c}
z \\
\bar{z} \\
u
\end{array}\right)= \\
& \left(\begin{array}{c}
z+t_{1} \frac{\partial z}{\partial z}+\frac{t_{1}^{2}}{2 !}\left(\frac{\partial}{\partial z}\left(\frac{\partial z}{\partial z}\right)\right)+\cdots \\
\bar{z}+t_{1} \frac{\partial \bar{z}}{\partial z}+\frac{t_{1}^{2}}{2 !}\left(\frac{\partial}{\partial z}\left(\frac{\partial \bar{z}}{\partial z}\right)\right)+\cdots \\
u+t_{1} \frac{\partial u}{\partial z}+\frac{t_{1}^{2}}{2 !}\left(\frac{\partial}{\partial z}\left(\frac{\partial u}{\partial z}\right)\right)+\cdots
\end{array}\right)=\left(\begin{array}{c}
z+t_{1} \\
\bar{z} \\
u
\end{array}\right) ; \\
& \left(\begin{array}{c}
z^{\prime} \\
\bar{z}^{\prime} \\
u^{\prime}
\end{array}\right)=\operatorname{Exp}\left(t_{3} Z_{3}\right)\left(\begin{array}{c}
z \\
\bar{z} \\
u
\end{array}\right)=\left(\begin{array}{c}
e^{2 t_{3}} z \\
\bar{z} \\
u+t_{3}
\end{array}\right) ; \\
& \left(\begin{array}{c}
z^{\prime} \\
\bar{z}^{\prime} \\
u^{\prime}
\end{array}\right)=\operatorname{Exp}\left(t_{5} Z_{5}\right)\left(\begin{array}{c}
z \\
\bar{z} \\
u
\end{array}\right)= \\
& =\left(\begin{array}{c}
\frac{z}{1-t_{5} z} \\
\bar{z} \\
u-\ln \left(1-t_{5} z\right)
\end{array}\right) .
\end{aligned}
$$

Применяя теперь экспоненциальное отображение (8) к базисным операторам $Z_{2}=\partial_{\bar{z}}, Z_{4}=$ $-2 \bar{z} \partial_{\bar{z}}+\alpha \partial_{u}$ и $Z_{6}=-\bar{z}^{2} \partial_{\bar{z}}+\alpha \bar{z} \partial_{u}$, аналогично получаем подгруппы:

$$
\begin{gathered}
z^{\prime}=z, \bar{z}^{\prime}=\bar{z}+t_{2}, u^{\prime}=u \\
z^{\prime}=z, \bar{z}^{\prime}=e^{-2 t_{4}} \bar{z}, u^{\prime}=u+\alpha t_{4} ; \\
z^{\prime}=z, \bar{z}^{\prime}=\frac{\bar{z}}{1+t_{6} \bar{z}}, u^{\prime}=u+\alpha \ln \left(1+t_{6} \bar{z}\right) .
\end{gathered}
$$

Из симметрии операторов $Z_{1}, Z_{3}, Z_{5}$ и $Z_{2}, Z_{4}$, $Z_{6}$ вытекает симметрия_для выражений найденных подгрупп, т. е. $\bar{z}^{\prime}=z^{\prime}$, поэтому $t_{2}=\bar{t}_{1}$, $t_{4}=-\bar{t}_{3}, t_{6}=-\bar{t}_{5}$. С учетом полученного, вычисляя композицию выше найденных подгрупп, имеem

$$
\begin{aligned}
z^{\prime}= & \frac{a z+b}{c z+d}, \\
& u^{\prime}=u+\alpha \ln \overline{(c z+d)}-\ln (c z+d)+p,
\end{aligned}
$$

причем $a=e^{2 t_{3}} \neq 0, b=t_{1} e^{2 t_{3}}, c=-t_{5} e^{2 t_{3}}, d=$ $1-t_{1} t_{5} e^{2 t_{3}}=1+t_{1} c, p=t_{3}-\alpha \bar{t}_{3}, \triangle=a d-b c=e^{2 t_{3}} \neq 0$. В дробной формуле выражений (11) из свойства однородности следует, что каждый из параметров можно умножить на любое комплексное число, поэтому переходим к другим параметрам, которые обозначим соответственно $a^{\prime}=a / \beta, b^{\prime}=$ $b / \beta, c^{\prime}=c / \beta, d^{\prime}=d / \beta, p^{\prime}=p+\alpha \ln \bar{\beta}-\ln \beta$, где $\beta$ - это любое комплексное число, причем $\triangle^{\prime}=$ $a^{\prime} d^{\prime}-b^{\prime} c^{\prime} \neq 1$. Тогда формулы (11) примут вид:

$$
\begin{aligned}
& z^{\prime}=\frac{a^{\prime} z+b^{\prime}}{c^{\prime} z+d^{\prime}}, \\
& u^{\prime}=u+\alpha \ln \overline{\left(c^{\prime} z+d^{\prime}\right)}-\ln \left(c^{\prime} z+d^{\prime}\right)+p^{\prime},
\end{aligned}
$$

Воспользовавшись условием инвариантности функции пары точек относительно преобразований группы движений, т. е. равенством (5), получаем $\alpha \ln \Delta^{\prime}+2 p^{\prime}=0$. В (12) можно положить $\triangle^{\prime}=1$ (свойство однородности дробной формулы выражений (11)), $\beta$ - принимает подходящее значение, значит, $p^{\prime}=0$. Тогда группа движений собственно гельмгольцевой трехмерной геометрии в прежних обозначениях параметров принимает вид (9).

Аналогично находится группа движений симплициальной геометрии III типа - (10), в которых $u^{\prime}$ - мнимое число.

Заключение. В работе представлено полное решение задачи о нахождении групп движений для собственно гельмгольцевой и симплициальной III типа трехмерных геометрий методом экспоненциального отображения. Эта задача может быть продолжена на особые феноменологически симметричные расширения евклидовых и псевдоевклидовых геометрий размерности выше 3 [10].

Остаются актуальными также задачи о разработке методов решения функциональных уравнений на множество движений [11], методов вывода уравнения феноменологической симметрии (ФС) трехмерных геометрий [12].

\section{Библиографический список}

1. Лев В.Х. Трехмерные геометрии в теории физических структур // Вычислительные системы. Новосибирск, 1988. Вып. 125.

2. Кыров В.А., Богданова Р.А. Группы движений некоторых трехмерных геометрий максимальной подвижности //Сиб. матем. журн. 2018. Т. 59, № 2. C. 412-421; Siberian Math. J., 59:2 (2018), 323-331. DOI:10.17377/smzh(2018)59.215.
3. Бредон Г. Введение в теорию компактных групп преобразований. М., 1980.

4. Понтрягин Л.С. Непрерывные группы. М., 1973.

5. Овсянников Л.В. Групповой анализ дифференциальных уравнений. М., 1978.

6. Кыров В.А. Шестимерные алгебры Ли групп движений трехмерных феноменологически 
симметричных геометрий // Приложение к книге Михайличенко Г.Г. «Полиметрические геометрии». Новосибирск, 2001.

7. Ляховский В.Д., Блохов А.А. Группы симметрии и элементарные частицы. Л., 1983.

8. Новиков С.П., Тайманов И.А. Современнные геомтерические структуры и поля. М., 2005.

9. Постников М.М. Группы и алгебры Ли. М., 1982.

10. Кыров В.А., Михайличенко Г.Г. Аналитический метод вложения евклидовой и псевдоевклидовой геометрий // Тр. ИММ УрО РАН. 2017. Т. 23, № 2.
11. Богданова Р.А. Группы движений двумерных гельмгольцевых геометрий как решение функционального уравнения // Сиб. журн. индустр. матем. 2009. Т. 12, № 4 .

12. Богданова Р.А., Михайличенко Г.Г. Вывод уравнения феноменологической симметрии для некоторых трехмерных геометрий // Изв. вузов. Матем.2018. T. 62, № 9. C. 11-20; Russian Math. (Iz. VUZ), 62:9 (2018), 7-16. DOI:10.3103/S1066369X18090025. 